\title{
Monitoring synaptic transmission in primary neuronal cultures using local extracellular stimulation
}

\author{
Anton Maximov ${ }^{\mathrm{a}, \mathrm{b}, 1}$, Zhiping P. Pang ${ }^{\mathrm{a}, \mathrm{b}, 1}$, Dougal G.R. Tervo ${ }^{\mathrm{a}, \mathrm{b}, \mathrm{c}}$, Thomas C. Südhof ${ }^{\mathrm{a}, \mathrm{b}, *}$ \\ ${ }^{a}$ Center for Basic Neuroscience, Department of Molecular Genetics, 6000 Harry Hines Blvd. Dallas, TX 75390-9111, USA \\ ${ }^{\mathrm{b}}$ Howard Hughes Medical Institute, UT Southwestern Medical Center, 6000 Harry Hines Blvd. Dallas, TX 75390-9111, USA \\ ${ }^{\mathrm{c}}$ Watson School of Biological Sciences, Cold Spring Harbor Laboratory, 1 Bungtown Road, Cold Spring Harbor, New York, NY 11724, USA
}

Received 7 September 2006; received in revised form 10 October 2006; accepted 11 October 2006

\begin{abstract}
Various techniques have been applied for the functional analysis of synaptic transmission in cultured neurons. Here, we describe a method of studying synaptic transmission in neurons cultured at high-density from different brain regions such as the cortex, striatum and spinal cord. We use postsynaptic whole-cell recordings to monitor synaptic currents triggered by presynaptic action potentials that are induced by brief stimulations with a nearby extracellular bipolar electrode. Pharmacologically isolated excitatory or inhibitory postsynaptic currents can be reliably induced, with amplitudes, synaptic charge transfers, and short-term plasticity properties that are reproducible from culture to culture. We show that the size and kinetics of pharmacologically isolated inhibitory postsynaptic currents triggered by single action potentials or stimulus trains depend on the Ca ${ }^{2+}$ concentration, temperature and stimulation frequency. This method can be applied to study synaptic transmission in wildtype neurons infected with lentiviruses encoding various components of presynaptic release machinery, or in neurons from genetically modified mice, for example neurons carrying floxed genes in which gene expression can be acutely ablated by expression of Cre recombinase. The preparation described in this paper should be useful for analysis of synaptic transmission in inter-neuronal synapses formed by different types of neurons.
\end{abstract}

(C) 2006 Elsevier B.V. All rights reserved.

Keywords: Synaptic transmission; Neuronal culture; GABA; AMPA; Extracellular stimulation

\section{Introduction}

Primary neuronal cultures have been extensively used for the characterization of the functional properties of synapses and for studies of the synaptic effects of pharmacological agents, expressed proteins, and genetic modifications. In studying synaptic transmission, the development of whole-cell voltageclamp techniques with a high resolution was instrumental (Hamill et al., 1981), as was the availability of specific receptor blockers that enabled monitoring physiologically and pharmacologically isolated postsynaptic currents.

In cultured neurons, two principal approaches have traditionally been used to analyze synaptic transmission: recordings from mixed populations of neurons that are cultured at relatively high density and form inter-neuronal synapses, and recordings from isolated neurons that form autapses. These approaches dif-

\footnotetext{
* Corresponding author. Tel.: +1 214648 1876; fax: +1 2146481879 .

E-mail address: Thomas.Sudhof@UTSouthwestern.edu (T.C. Südhof).

1 These authors contributed equally to the study.
}

fer in how presynaptic inputs are activated, but share the same fundamental postsynaptic voltage-clamp recording technique.

In recordings from mixed populations of neurons, presynaptic inputs can be stimulated by several techniques. These include: (1) the localized application of electrical pulses (Kirischuk et al., 1999; Kirischuk and Grantyn, 2003; Chen et al., 2004) or of a solution containing elevated $\mathrm{K}^{+}$(Liu and Tsien, 1995a,b) to individual synapses that are visualized with fluorescent dye, (2) the depolarization of patched presynaptic neurons in a pairedrecording configuration (Bi and Poo, 1998), or (3) electrical field stimulations that activate all neurons on a plate (Schoch et al., 2001; Deak et al., 2004).

In all of these recordings of synaptic transmission from mixed high-density cultures, pre- and postynaptic elements are being analyzed that are formed by separate neurons, inter-neuronal synapses are examined. In contrast, in recordings of synaptic transmission in autapses, pre-and postsynaptic elements are studied that are derived from the same single isolated neuron. Specifically, neurons are induced to form autapses by culturing them on microislands of glia cells, and are analyzed with a single whole-cell pipette that serves both as the presynaptic stimulation 
electrode and as the postsynaptic recording electrode (Bekkers and Stevens, 1991; Fernandez-Chacon et al., 2001; Pyott and Rosenmund, 2002; Otsu et al., 2004). The recording configuration in autapses enables monitoring of synaptic transmission between well-defined pre-and postsynaptic inputs, and makes it possible to achieve quantitative reproducible recordings of synaptic transmission. In contrast, in mixed high-density cultures of neurons it is difficult to achieve presynaptic stimulations that are reproducible from culture to culture, and thus difficult to quantitate synaptic parameters. For example, although focal stimulations allow a kinetic analysis of pre- and postsynaptic parameters of single synaptic boutons at high resolution, variations in the size of postsynaptic currents between individual synapses complicate quantitative analysis of release (Liu and Tsien, 1995a). Paired recordings provide a valuable tool for studying synaptic transmission between well-defined pairs of synaptically connected neurons (Bi and Poo, 1998), but are labor-intensive, and cannot be easily standardized because the number of synaptic connections between neurons varies (Maximov and Südhof, unpublished observations). Field-stimulations may be the simplest and most reproducible method of presynaptic stimulation, but the stimuli are not homogeneous across the field, are not suitable for high-resolution analyses, and often are so large that they can lead to significant artifacts.

Although autaptic microisland cultures have many advantages over mixed high-density cultures of neurons for recording purposes, autapses also exhibit disadvantages. It is unlikely that autapses are physiologically identical to 'normal' synapses formed between different pre- and postsynaptic neurons. Indeed, analyses of synaptic transmission in neurons lacking the calcium sensor for synaptic vesicle exocytosis, synaptotagmin-1, performed in autaptic or mixed cultures resulted in differences between spontaneous release phenotypes (Geppert et al., 1994; Maximov and Südhof, 2005; Pang et al., 2006a). Furthermore, synaptic plasticity is difficult to analyze in autapses. Moreover, synaptic responses in autapses also differ dramatically from culture to culture, a finding that is counterintuitive considering the fact that in autapses one would expect neurons to form the same number of synaptic contacts since a variation of culture density is not an issue.

In this study, we describe an approach to analyze inhibitory and excitatory synaptic transmission triggered in mixed highdensity cultures of neurons that uses well-established methods to avoid some of the problems mentioned above. We employ local stimulation of presynaptic inputs via bipolar electrodes that are placed near the soma of the postsynaptic neuron that is being analyzed. We show that such stimulations reliably evoke neurotransmitter release by eliciting action potentials in synaptic inputs formed by multiple axons from neighboring presynaptic cells. The size of the synaptic responses is reproducible from culture to culture. We demonstrate that the magnitude of pharmacologically isolated GABAergic inhibitory postsynaptic currents (IPSCs) triggered by isolated stimuli depends on the number of activated presynaptic inputs, the temperature, and the concentration of extracellular calcium. We further describe that the kinetics of synaptic responses triggered by high-frequency stimulus trains depends on the concentration of free extracellular calcium, the rate of intracellular calcium buffering, the temperature and the stimulation frequency, suggesting that synapses triggered by extracellular stimulation display normal calcium sensitivity and short-term plasticity. Our data indicate that stimulation via local bipolar electrodes enables the monitoring of synaptic transmission in cultured neurons in near-physiological conditions. We recently employed this method to study the regulation of neurotransmitter release in wildtype neurons infected with lentiviruses expressing various components of the synaptic vesicle release machinery (Tang et al., 2006), and in neurons from genetically modified mice (Maximov and Südhof, 2005; Ho et al., submitted for publication; Pang et al., 2006b).

\section{Materials and methods}

\subsection{Neuron culture}

Cells in the spinal cord of E13.5-14.5 mouse pups, or the cortex, hippocampus, or striatum of E18 or P0 mouse or rat pups were dissociated by incubation for $7 \mathrm{~min}$ at $37^{\circ} \mathrm{C}$ in digestion solution containing $6 \mathrm{mg} / \mathrm{ml}$ trypsin (Sigma, Cat\# T1005-1G), $0.5 \mathrm{mg} / \mathrm{ml}$ DNAse (Sigma, Cat\# D5025) and (in mM) 137 $\mathrm{NaCl}, 5 \mathrm{KCl}, 7 \mathrm{Na}_{2} \mathrm{HPO}_{4}$, and $25 \mathrm{HEPES}-\mathrm{NaOH} \mathrm{pH}$ 7.2. The dissociated cells containing neurons were then washed once with Hank's balanced salt solution (HBS) containing 20\% fetal bovine serum (FBS) followed by two washes in serum-free HBS, and further dissociated by gentle pipetting in HBS containing $12 \mathrm{mM} \mathrm{MgSO}_{4}$ and $0.5 \mathrm{mg} / \mathrm{ml}$ DNAse. The cell suspension was centrifuged for $10 \mathrm{~min}$ at $160 \mathrm{~g}$ and plated on Matrigel (Collaborative Biomedical Products, Cat\# 871-275-0004) coated circular glass coverslips ( with B27 (Invitrogen, Cat\# 17504-044), glucose, transferrin and $5 \%$ fetal bovine serum. For cortical cultures, the cell suspension obtained from the cortex of a single brain was used to plate 12 wells in a 24-well plate. For striatal cultures, the cell suspension obtained from striatum of a single brain was used to plate 3 wells. For spinal cord culture, ventral horn stripes from thoracic to lumber segments were used to plate 12 wells. For all cultures, the initial cell density (including glia) at plating varied between 1500 and $2500 \mathrm{~mm}^{-2}$. When the density of glia cells in the culture reached $\sim 40-50 \%$ (usually 2 days after plating), $50 \%$ of the conditioned culture medium was replaced with fresh medium containing $4 \mathrm{mM}$ Ara-C (Sigma). The cultures were maintained in medium containing $2 \mathrm{mM}$ Ara-C until experiments (13-18 DIV). The described procedure can also be used for older pups (P1-P3); however, we found that survival of neurons in culture progressively decreases with the age of animals.

\subsection{Electrophysiology}

In the standard protocol, a neuron intended for postsynaptic recordings was patched with pipettes that were pulled from borsilicate glass capillary tubes (Warner Instruments, Cat\# 640793) using a PP830 pipette puller (Narishige). The whole-cell pipette solution contained (in $\mathrm{mM}$ ) $135 \mathrm{CsCl}, 10$ HEPES, 1 EGTA, $1 \mathrm{Na}-\mathrm{GTP}, 4 \mathrm{Mg}$-ATP and 10 QX-314 (pH 7.4, adjusted with $\mathrm{CsOH}$ ). The resistance of pipettes filled with the intra- 
cellular solution varied between 4 and $5 \mathrm{M} \Omega$. After formation of the whole-cell configuration and equilibration of the intracellular pipette solution, the series resistance was adjusted to $10 \mathrm{M} \Omega$. Cells whose initial $R_{\mathrm{s}}$ exceeded $15 \mathrm{M} \Omega$ were excluded from analysis. Synaptic currents were monitored with Multiclamp 700 A amplifier (Molecular Devices). Presynaptic inputs were stimulated with a bipolar electrode (FHC; www.fh-co.com; Cat\# CBAEC75-Concentric Bipolar Electrode OP: $125 \mu \mathrm{m} \mathrm{SS}$; IP: $25 \mu \mathrm{m} \mathrm{Pt} / \mathrm{l})$ that was placed nearby the patched neuron and used to apply a $0.9 \mathrm{~mA}, 1 \mathrm{~ms}$ current injection. The distance between the tip of stimulation electrode and the cell body of postsynaptic neuron varied between 100 and $150 \mu \mathrm{m}$. This variability had no dramatic effect on the sizes of evoked responses. In experiments shown in Fig. 3, evoked responses were triggered by stimulations of variable intensity and constant duration $(0.1-1.5 \mathrm{~mA} / 1 \mathrm{~ms})$ or constant intensity and variable duration (0.9 mA/0.1-1 ms). The frequency, duration, and magnitude of the extracellular stimulus were controlled with a Model 2100 Isolated Pulse Stimulator (A-M Systems, Inc.) synchronized with Clampex 9 data acquisition software (Molecular Devices). The bath solution contained (in $\mathrm{mM}$ ) $140 \mathrm{NaCl}, 5 \mathrm{KCl}, 0.8$

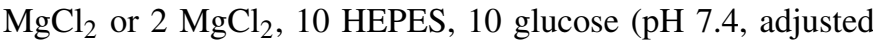
with $\mathrm{NaOH}$ ) and various concentrations of free extracellular calcium. Inhibitory and excitatory postsynaptic currents were pharmacologically isolated by adding AMPA and NMDA receptor blockers CNQX $(20 \mu \mathrm{M})$ and APV $(50 \mu \mathrm{M})$ or GABA $_{A}$ receptor blockers bicucullin $(20 \mu \mathrm{M})$ or picrotoxin $(50 \mu \mathrm{M})$ to the extracellular bath solution. Spontaneous miniature inhibitory postsynaptic currents (mIPSCs) were monitored in the presence of tetrodotoxin $(1 \mu \mathrm{M})$ to block action potentials. The temperature in the recording chamber was controlled by TC344B dual temperature controller (Warner Instruments).

\subsection{Data analysis}

Postsynaptic currents were sampled at $10 \mathrm{kHz}$ and analyzed off-line using Clampfit 9 (Molecular Devices) and Origin7 software (Mocrocal Inc.). For graphic representation, the current traces shown in Figs. 6A, 7E and 8G and 9B were filtered by $50 \mathrm{~Hz}$ low-pass Gaussian filter to remove the stimulus artifacts. The amplitudes of NMDA EPSCs recorded at $+40 \mathrm{mV}$ were calculated at $50 \mathrm{~ms}$ after stimulus application to rule out contamination with outward AMPA currents. To measure the frequency of spontaneous release or amplitudes of synchronous IPSCs during the stimulus trains, individual mIPSCs or IPSCs were collected using the pClamp template search function. The time constants for equilibration of pipette solution were determined by approximating the plots of mIPSC frequencies or IPSC amplitudes as a function of time after break-in to single exponential fit $\left(y=y_{0}+A_{1} \times \exp \left(-\left(x-x_{0}\right) / \tau_{1}\right)\right.$, where $y$ is the mIPSC frequency or IPSC amplitude, $x$ the time after break in and $\tau_{1}$ is the equilibration time constant. The time constants of single evoked IPSCs were determined by approximating the cumulative charge histograms to a double exponential fit $\quad\left(y=y_{0}+A_{1} \times \exp \left(-\left(x-x_{0}\right) / \tau_{1}\right)+A_{2} \times \exp \left(-\left(x-x_{0}\right) / \tau_{2}\right)\right.$ where $y$ is the normalized cumulative charge transferred by single IPSC, $x$ the time after stimulation and $\tau_{1}$ and $\tau_{2}$ are the time constants for fast and slow components. The apparent affinity for extracellular $\mathrm{Ca}^{2+}$, was determined by approximating the IPSC dose-response plot with the Hill function $\left(I=I_{\max } /\left(1+\left(K_{\mathrm{d}} /\left[\mathrm{Ca}^{2+}\right]\right)^{n}\right)\right.$ where $I$ is the amplitude of synaptic current, $I_{\max }$ the maximal amplitude, $K_{\mathrm{d}}$ the apparent dissociation constant for extracellular $\mathrm{Ca}^{2+},\left[\mathrm{Ca}^{2+}\right]$ the extracellular $\mathrm{Ca}^{2+}$ concentration, and $n$ the apparent cooperativity. Statistical analysis was performed with Student's $t$-test $\left({ }^{*} P<0.001\right)$. All data shown are means \pm S.E.M.s.

\section{Results}

\subsection{Monitoring evoked excitatory and inhibitory postsynaptic currents}

We prepared mixed cell cultures from the cortex, hippocampus, and striatum of embryonic E18 or postnatal P0 rat or mouse pups or spinal cords of embryonic E13.5-14.5 mouse pups. The cells were dissociated by trypsin digestion, plated at relatively high density $\left(1500-2500 \mathrm{~mm}^{-2}\right)$ on Matrigel-coated circular glass coverslips, and cultured for 13-18 days. At this stage in culture, neurons develop a fully polarized phenotype with extensive synaptic networks (Fig. 1A and data not shown), and display robust postsynaptic responses (see below). We used whole-cell recordings to monitor synaptic currents in response to presynaptic action potentials. The technique described here to induce presynaptic action potentials was developed because other stimulation methods had multiple disadvantages, prompting us to explore the use of stimulations by a local electrode that is placed at a defined distance from the patched postsynaptic neuron. The stimulating electrode was placed into the recording chamber near the upper surface of the glass coverslip with the attached neurons within $100-150 \mu \mathrm{m}$ from the soma of the patched neuron (Fig. 1A).

To monitor inhibitory postsynaptic currents (IPSCs), we used a pipette solution containing $135 \mathrm{mM} \mathrm{Cl}^{-}$as a charge carrier and $10 \mathrm{mM}$ QX314 as a blocker of sodium currents in the postsynaptic cells, and maintained the holding potential at $-70 \mathrm{mV}$. Excitatory AMPA and NMDA receptors were blocked by adding CNQX $(20 \mu \mathrm{M})$ and APV $(50 \mu \mathrm{M})$ to the extracellular bath solution. Under these conditions, IPSCs manifested as inward currents (Fig. 1B and E). We found that brief injections of current $(0.9 \mathrm{~mA}$ for $1 \mathrm{~ms})$ through the stimulating electrode induced robust IPSCs in neurons isolated from cortex, striatum and spinal cord (Fig. 1B, D and E). The IPSCs immediately followed the stimulus application and could be completely abolished by bath application of the sodium channel blocker tetrodotoxin $(1 \mu \mathrm{M})$, suggesting that postsynaptic responses were elicited by action potentials that trigger neurotransmitter release in presynaptic terminals contacting the patched neuron (Fig. 1B). In cortical cultures, the IPSCs were completely suppressed by extracellular application of GABA receptor blocker bicucullin $(20 \mu \mathrm{M})$, indicating that in these neurons postsynaptic responses were exclusively induced by $\mathrm{GABA}_{\mathrm{A}}$ receptors and were not contaminated with other types of postsynaptic currents (Fig. 1B). In contrast, in neurons isolated from the spinal cord, postsynaptic responses were only partially blocked by bicucullin but were 
(A)

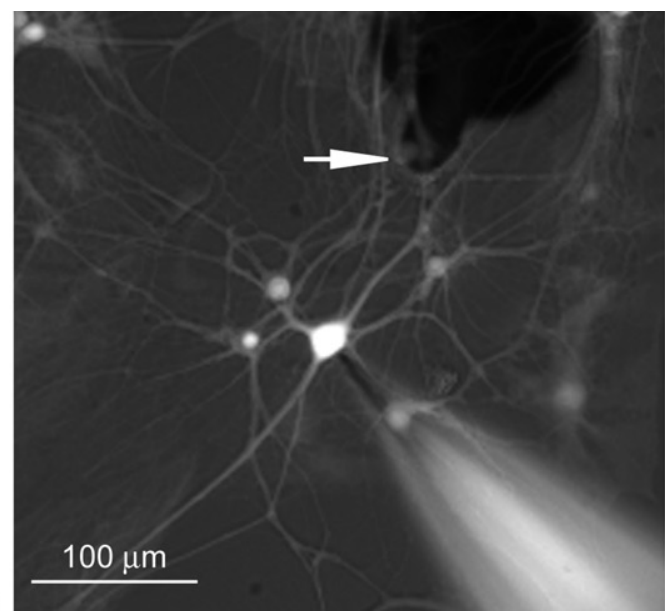

completely suppressed by bicucullin combined with strychnine $(10 \mu \mathrm{M})$, suggesting that in these neurons IPSCs are mediated by both $\mathrm{GABA}_{\mathrm{A}}$ and glycine receptors (Fig. 1E).

To monitor evoked excitatory postsynaptic currents (EPSCs), we performed recordings from cortical neurons maintained at $-70 \mathrm{mV}$ in a bath solution containing GABA receptor blocker picrotoxin (PTX, $50 \mu \mathrm{M})$. Under these conditions, stimulations with the local electrode induced inward currents with a time course typical for fast AMPA-type EPSCs (Fig 1C). Since analysis of excitatory transmission was complicated by occasional network activity (see discussion below in Section 3.7), we first focused on the characterization of inhibitory synaptic transmission. We analyzed cortical neuronal cultures because these cultures can be easily prepared in relatively large scale from embryonic or postnatal animals, although the results similarly apply to neurons prepared from the hippocampus (data not shown). The preparation described here can be applied to study synaptic transmission in wildtype neurons infected with lentiviruses encoding various components of presynaptic release machinery (Tang et al., 2006), or in neurons from genetically modified mice, for example neurons carrying floxed genes in which gene expression can be acutely ablated by expression of Cre recombinase (Ho et al., submitted for publication).

\subsection{Parameters for whole-cell recording of postsynaptic currents}

Measurements of synaptic currents using whole-cell recordings can be affected by several artifacts, including limited diffusion of the pipette solution into the cell resulting in variability in intracellular concentration of the charge carrier $\left(\mathrm{Cl}^{-}\right)$, incomplete blockage of voltage-dependent sodium channels that may contaminate postsynaptic responses, changes in the series resistance $\left(R_{\mathrm{S}}\right)$ during recording, poor space clamp, and stimulus artifacts that may overlap with the initial phase of IPSCs and would make it difficult to analyze the IPSC amplitudes and the rise times.

\subsubsection{Equilibration after break-in and block of sodium channels}

To estimate the rate of equilibration of the pipette solution with the cytosol, we recorded spontaneous miniature IPSCs

\footnotetext{
blocker bicucullin (BIC, lower trace), indicating that IPSCs are elicited by action potentials induced in axons nearby, and are not contaminated with other types of currents. Scale bars apply to all traces. In this and panels C-E, postsynaptic currents were triggered by $0.9 \mathrm{~mA}$ extracellular stimulations applied for $1 \mathrm{~ms}$ via a local bipolar electrode and recorded in whole-cell mode. QX314 (10 mM) was added to the pipette solution to block the sodium currents in postsynaptic neurons. Arrows indicate the times of stimulations. (C) Excitatory AMPA-type postsynaptic currents (EPSCs) recorded from cortical neurons in the presence of GABA receptor blocker picrotoxin (PTX, $50 \mu \mathrm{M})$. (D-E) Typical IPSCs monitored in the presence of CNQX $(20 \mu \mathrm{M})$ and APV $(50 \mu \mathrm{M})$ from cultured neurons isolated from striatum (D) and spinal cord (E). Note that in spinal cord cultures, postsynaptic currents are only partially blocked by bicuculline (BIC), indicating that IPSCs in these cultures are mediated by both GABA and glycine receptors. Glycinergic currents recorded in the presence of bicucullin could be completely blocked by $10 \mu \mathrm{M}$ strychnine (BIC + STR).
} 


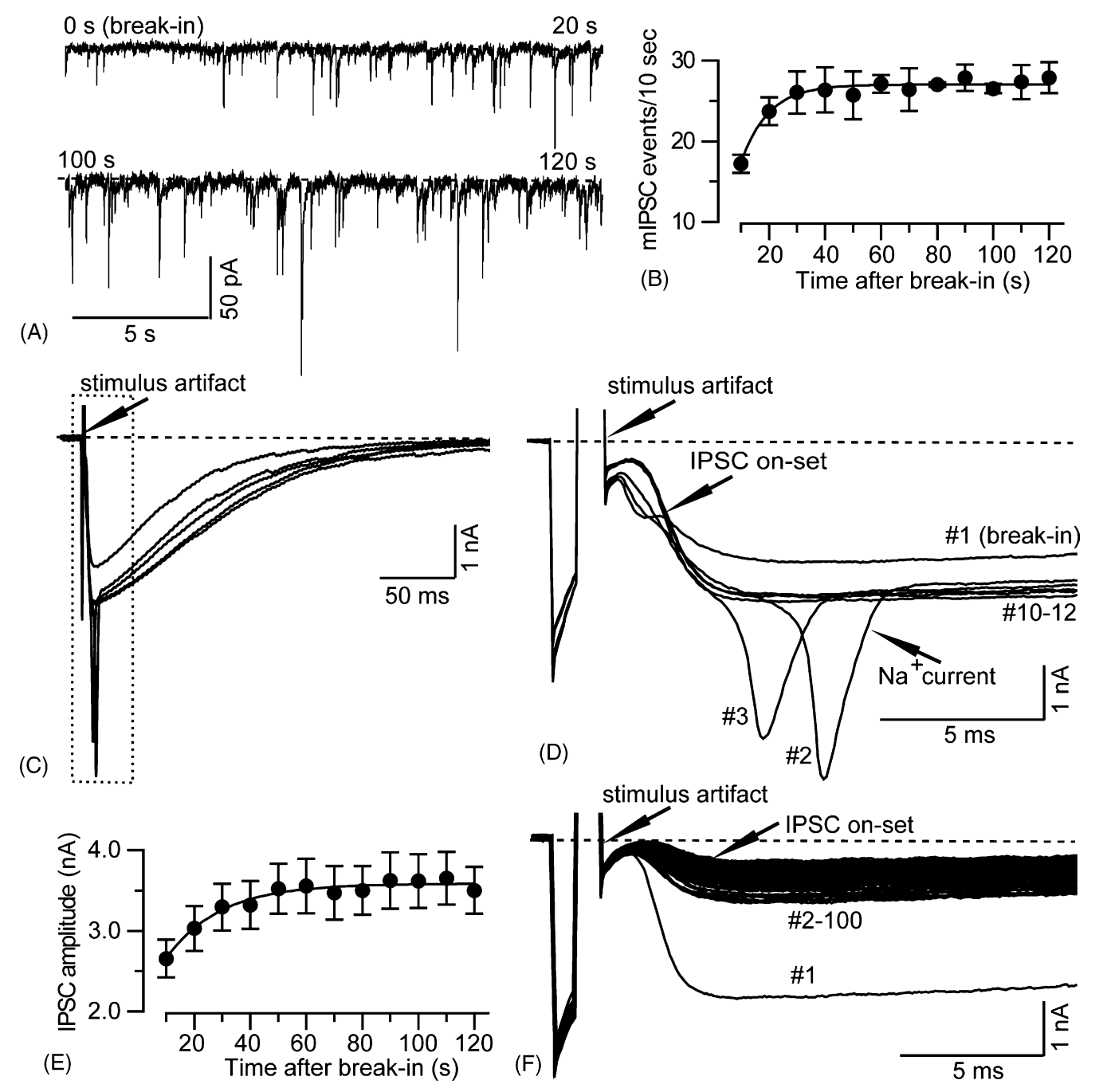

Fig. 2. Spontaneous and evoked inhibitory responses reach steady-state levels during the first minute after formation of the whole-cell patch (i.e., after break-in). (A) Typical examples of spontaneous miniature IPSCs (mIPSCs) monitored from cortical neurons for $20 \mathrm{~s}$ immediately after break-in (top trace) and $100 \mathrm{~s}$ after break-in (bottom trace). Scale bars apply to both traces. (B) Average numbers of individual mIPSC events monitored from three different neurons for every $10 \mathrm{~s}$ after break-in plotted as a function of time and approximated to a single exponential fit (solid curve; time constant $=21 \mathrm{~s}$ ). (C) Evoked IPSCs triggered by $0.1 \mathrm{~Hz}$ stimulations and recorded immediately after break-in. Arrow indicates the stimulus artifact. Dotted box shows the area expanded in panel D. (D) Expanded initial segments of evoked IPSCs\# 1-3 and 10-12 of the experiment shown in panel C (dotted box). Arrows indicate the stimulus artifacts, IPSC on-set, and sodium currents that occasionally contaminate the first IPSCs after break-in. Note that postsynaptic currents progressively increase in size reaching a plateau during first minute after break-in. (E) Average amplitudes of IPSCs monitored at $0.1 \mathrm{~Hz}$ from 12 different neurons were plotted as a function of time after break-in. IPSCs amplitudes are shown as absolute values; data were approximated to a single exponential fit (solid curve; time constant $=29 \mathrm{~s}$ ). (F) Expanded initial segments of evoked IPSCs triggered by $10 \mathrm{~Hz}$ stimulus train applied for $10 \mathrm{~s}$. Note that the shapes of stimulus artifacts (marked by arrows) do not change during the high-frequency trains.

(mIPSCs) immediately after formation of whole-cell configuration on the postsynaptic neuron (break-in). We found that the frequency of spontaneous mIPSC events progressively increased after break-in, reaching a plateau after $\sim 40 \mathrm{~s}$ (Fig. 2A and B). Similar to the spontaneous events, we found that evoked IPSCs monitored at $0.1 \mathrm{~Hz}$ progressively increased in size after breakin, also reaching a plateau after $\sim 40 \mathrm{~s}$ (Fig. 2C-E). The first several postsynaptic responses observed after break-in were frequently contaminated with sodium currents, most likely because the concentration of QX314 inside the cells was too low to completely block the $\mathrm{Na}^{+}$channels. In contrast, no contamination with sodium currents was observed when IPSCs were monitored after 1-2 min upon formation of the whole-cell patch (Fig. 2D).
Thus, we always allowed a 2 min delay after break-in before collecting the data to measure the frequency and the amplitudes of spontaneous mIPSCs and the amplitudes of evoked IPSCs.

\subsubsection{Series resistance $\left(R_{S}\right)$ during recording, space clamp problems and stimulus artifacts}

Common whole-cell recording problems can arise from several sources. First, a poor space clamp can arise from a poor voltage clamp at the soma, which is frequently caused by poor break-in or an inadequate series resistance compensation. We tested if the series resistance contributed to the measured amplitude of synaptic current by testing for a correlation between the $R_{\mathrm{S}}$ and the amplitude of evoked responses across cells. We found 
a slight but significant correlation between these parameters ( $r^{2}=0.26$; data not shown) for $R_{\mathrm{S}}$ below $15 \mathrm{M} \Omega$. For this reason we culled all cells whose initial $R_{\mathrm{S}}$ exceeded $15 \mathrm{M} \Omega$ and compensated $R_{\mathrm{S}}$ to $10 \mathrm{M} \Omega$ prior recordings. Second, local changes in the membrane potential caused by, for example, synaptic input can diminish the driving force at eletrotonically near-by synapses and thus lead to an underestimate of evoked IPSCs recorded from the cell body. Given the relatively large size of the evoked IPSCs, we cannot eliminate the possibility that this effect exists, and it is particularly important to take into consideration when trying to interpret trains of evoked currents. Third, space clamp errors can arise if the measured synaptic inputs are electronically distant from the clamped cell body. Although this problem most likely exists given that in the mature cultured neurons GABAergic synapses are uniformly distributed across the large dendritic trees (Swanwick et al., 2006), the fact that saturating stimulation intensities were used to evoke IPSCs may prevent any systematic bias. In order to determine if a systematic bias exists, we tested if there was a correlation between the risetime (19-90\%) and decay constant of recorded currents. We did not find a significant correlation between these parameters $\left(r^{2}=-0.04\right.$; data not shown). Although significant errors may arise from a poor space clamp in these recordings and lead to an underestimate of the amplitude of evoked IPSCs, these errors do not undermine the comparison of the amplitude of evoked currents in different conditions. We also found that the stimulus artifacts produced by $0.9 \mathrm{~mA} / 1 \mathrm{~ms}$ stimulations did not overlap with IPSC on-sets (Fig. 2D) and were constant in shape during the trains of action potentials, indicating that the series resistance $\left(R_{\mathrm{S}}\right)$ of postsynaptic neurons does not change during the high-frequency stimulus trains (Fig. $2 \mathrm{E}$ and F).

\subsection{Optimization of parameters for extracellular stimulation}

To establish the relationship between the sizes of postsynaptic responses and parameters of extracellular stimulation, we next measured the size of the IPSCs as a function of stimulus intensity (Fig. 3A and B) or stimulus duration (Fig. 3C and D). We found that the IPSC amplitude and charge induced by $1 \mathrm{~ms}$ stimulations with increasing intensity rose with the stimulus strength, reaching a plateau at a stimulus intensity of $\sim 0.8 \mathrm{~mA}$. IPSCs triggered by low-intensity stimulations $(0.1-0.6 \mathrm{~mA})$ varied significantly from trial to trial, as evidenced by the high coefficient of variation under these conditions (Fig. 3A and B, inserts), indicating that multiple presynaptic inputs formed by several axons contribute to the integrated postsynaptic responses, and that stimulation intensities of $<0.8 \mathrm{~mA}$ failed to trigger action potentials in some of these axons. A similar relationship was observed when IPSCs were induced by a constant $0.9 \mathrm{~mA}$ current injection for various durations (Fig. $3 \mathrm{C}$ and D). Since $0.9 \mathrm{~mA} / 1 \mathrm{~ms}$ stimulations evoked robust IPSCs without failures and stimulations of longer durations or higher intensities produced larger stimulus artifacts and sometimes resulted in electrolysis of the electrode tip, we choose $0.9 \mathrm{~mA}$ stimulations for $1 \mathrm{~ms}$ for all our experiments.

\subsection{Reproducibility of inhibitory synaptic currents monitored in different cortical culture preparations}

The peak amplitudes of IPSCs triggered by $0.9 \mathrm{~mA} / 1 \mathrm{~ms}$ stimulations and monitored from 167 wildtype neurons cultured in vitro for 13-18 days varied from 1 to $10 \mathrm{nA}$ with an average size of $3.5 \pm 0.2 \mathrm{nA}$ (Fig. 4A). Fluctuation in the sizes of IPSCs observed in different culture preparations can be a result of variability in cell and synaptic density, degree of synapse maturation, release probabilities of individual synapses and the number of presynaptic inputs activated in every trial. We found that the average amplitudes of IPSCs recorded on the same day after plating from 11 independent wildtype cortical cultures varied between $2.6 \pm 0.4$ and $4.5 \pm 0.4 \mathrm{nA}$ (Fig. 4B). In addition, a slight correlation between the sizes of postsynaptic responses and degree of culture maturation was observed. The amplitudes of IPSC recorded between 13 and 18 DIV were slightly larger in older, more mature neurons (data not shown). Thus, to minimize the variability caused by fluctuations in the cell density, synaptic density and synapse maturation, we always compared the sizes of synaptic responses using datasets collected from neurons that were analyzed after exactly the same number of days in culture.

\subsection{Calcium-dependence and short-term plasticity of inhibitory synapses in cortical cultures}

To ensure that the basic properties of synaptic transmission remain intact in cultured cortical neurons triggered by extracellular stimulation, we studied the calcium-dependence and short-term plasticity of inhibitory synapses. We measured the amplitudes of IPSCs evoked by single action potentials in the presence of different concentrations of free calcium in the extracellular bath solution (Katz and Miledi, 1968), and determined the rate of depression of IPSCs evoked by pairs of action potentials or stimulus trains applied at different frequencies (Figs. 5 and 6).

We found that the amplitudes of individual IPSCs triggered at low frequency $(0.1 \mathrm{~Hz})$ displayed a strong dependence on the concentration of free extracellular calcium (Fig. 5A). To calculate the apparent affinity for extracellular $\mathrm{Ca}^{2+}$, we fitted the dose-response plot with the Hill function $\left(I=I_{\max } /\right.$ $\left(1+\left(K_{\mathrm{d}} /\left[\mathrm{Ca}^{2+}\right]\right)^{n}\right)$, where $I$ is the amplitude of synaptic current, $I_{\max }$ the maximal amplitude, $K_{\mathrm{d}}$ the apparent dissociation constant for extracellular $\mathrm{Ca}^{2+},\left[\mathrm{Ca}^{2+}\right]$ the extracellular $\mathrm{Ca}^{2+}$ concentration, and $n$ the apparent cooperativity. The calculated $\mathrm{Ca}^{2+}$ affinity of inhibitory synapses in wildtype cultures was $1.8 \pm 0.2 \mathrm{mM}$, and the cooperativity was 2.9 . These data indicate that inhibitory synapses in cultured cortical neurons have similar calcium affinity of release compared to excitatory synapses of autaptic microisland cultures $\left(1.9 \pm 0.2 \mathrm{mM} \mathrm{Ca}^{2+}\right.$; FernandezChacon et al., 2001).

Residual accumulation of free intracellular calcium in presynaptic terminals stimulated with an action potential may increase the probability of vesicle release when the terminals are stimulated with the second action potential closely spaced with the first stimuli (Katz and Miledi, 1968). We next triggered the 

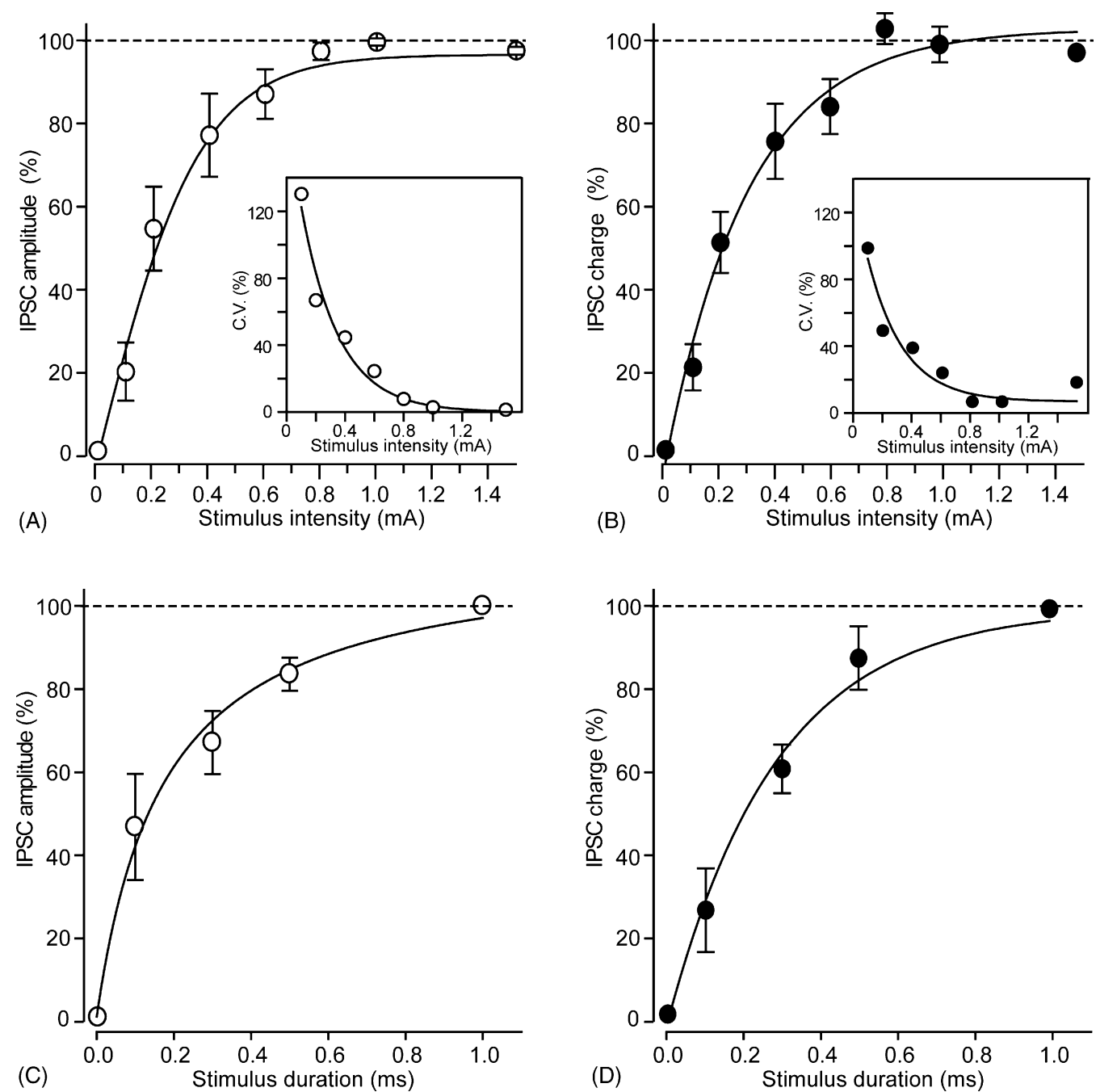

Fig. 3. Optimization of parameters for extracellular stimulation. IPSCs were triggered by action potentials elicited by low frequency $(0.1 \mathrm{~Hz})$ stimulations of constant duration and various intensities (A-B) or constant intensity and various durations (C-D). All recordings were performed in $2 \mathrm{mM}$ extracellular calcium. (A and B) Normalized amplitudes (A) and charge transfers (B) of IPSCs plotted as a function of stimulus intensity. IPSCs were triggered by $1 \mathrm{~ms}$ stimulations with $0.1-1.5 \mathrm{~mA}$. Responses are normalized for every neuron for the response observed with a $1.0 \mathrm{~mA}$ stimulus. Averaged data from 12 different neurons were approximated to a single exponential fit. Note that the size of postsynaptic response progressively increases, reaching a plateau at $\sim 0.8 \mathrm{~mA}$. The inserts show the plots of the coefficient of variance of the response as a function of stimulus intensity for responses triggered in different trials. Data were approximated to a single exponential fit. (C and $\mathrm{D}$ ) Normalized amplitudes (A) and charge transfers (B) of IPSCs plotted as a function of stimulus duration. IPSCs were triggered by $0.9 \mathrm{~mA}$ stimulations for $0.1-1.0 \mathrm{~ms}$. Data shown are normalized for the $1 \mathrm{~ms}$ responses. Averaged data from four different neurons were approximated to a single exponential fit (time constant $=0.3 \mathrm{~ms})$.

IPSCs by pairs of action potentials applied at different intervals and calculated the paired-pulse ratios of IPSC amplitudes as an indicator of release probability (Zucker and Regehr, 2002). We found that the paired-pulse ratio depended on the intervals between the first and the second stimuli and the concentration of extracellular calcium (Fig. 5B). Elevation of extracellular calcium enhanced paired-pulse depression; this effect was reversed by addition of membrane-permeable calcium chelator EGTA$\mathrm{AM}$, suggesting that the probability of neurotransmitter release triggered by extracellular stimulations depends on the amount of calcium influx (Fig. 5B). Consistent with this observation, we found that the rate of depression of synchronous IPSCs during the trains of action potentials depended on the stimulation frequency (Fig. 6). These results are in agreement with previously published observations on the properties of inhibitory synapses in cortical slices (Varela et al., 1999). Together our data suggest that inhibitory synapses triggered by local extracellular stimulation display normal calcium sensitivity and short-term plasticity.

\subsection{Effect of temperature on magnitude and kinetics of transmitter release in inhibitory synapses}

We next studied the effects of temperature on the sizes and kinetics of IPSCs triggered by low frequency stimulations or stimulus trains. Similarly to the previously reports in autapses (Pyott and Rosenmund, 2002), we found that the IPSCs monitored at near-physiological temperature $\left(35^{\circ} \mathrm{C}\right)$ in high-density cultures displayed larger amplitudes and faster kinetics of decay than the IPSCs recorded at room temperature (Fig. 7A-D). 

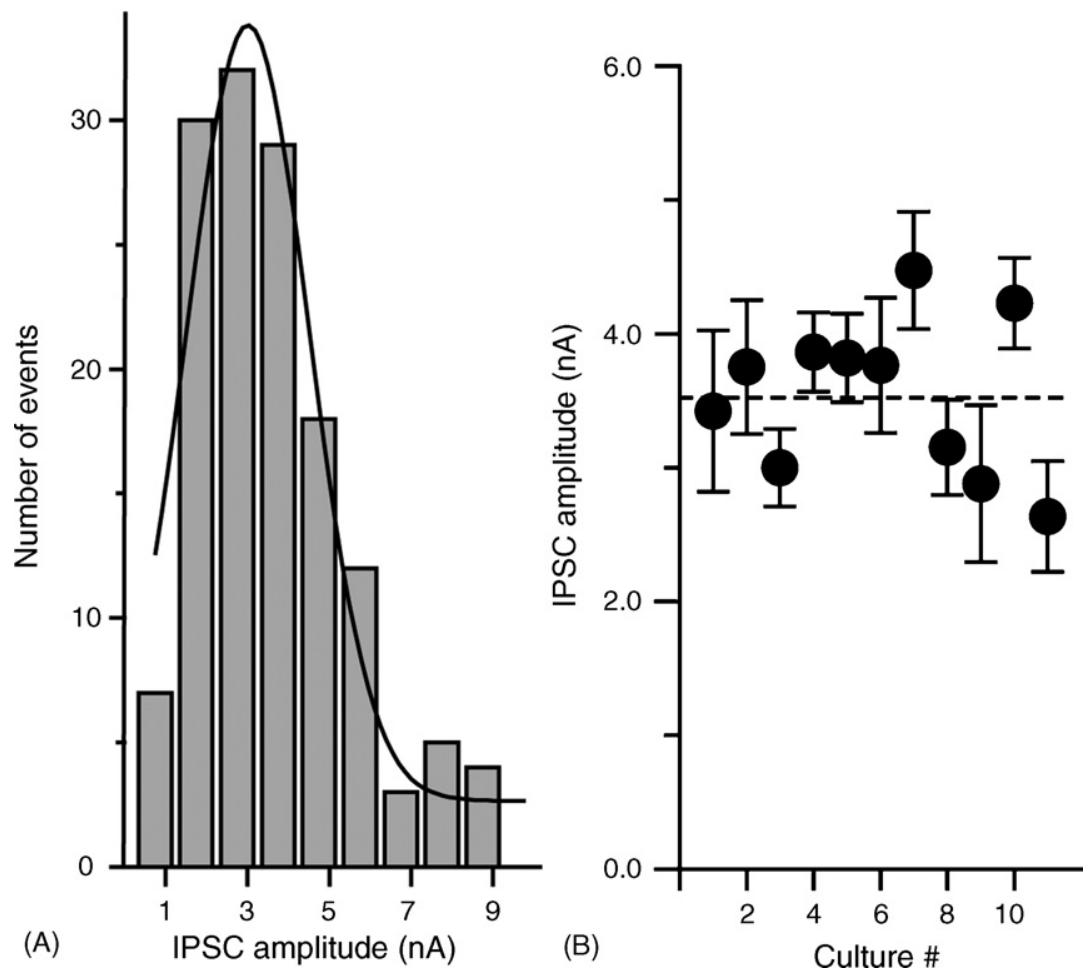

Fig. 4. Reproducibility of inhibitory synaptic currents monitored in different cortical culture preparations. (A) Histogram showing the distribution of amplitudes of IPSCs monitored from 167 wildtype cortical neurons in 12 independent cultures that were maintained in vitro for 13-18 days. Data are binned at $1 \mathrm{nA}$ intervals, and fitted to a Gaussian distribution (smooth curve) with a center at $3.5 \pm 0.2 \mathrm{nA}$. Action potentials were triggered by $0.9 \mathrm{~mA}$ stimulations applied for $1 \mathrm{~ms}$. (B) Average IPSC amplitudes determined in 11 different cultures or cortical neurons. For each culture, the data were collected on the same day between 14 and 17 DIV ( $n=5-14$ ). All recordings were performed in $2 \mathrm{mM}$ extracellular calcium (dashed line, mean amplitude).

Although the amplitudes of IPSCs recorded at $35^{\circ} \mathrm{C}$ were almost two-fold larger when compared to the IPSCs recorded at $25^{\circ} \mathrm{C}$, no significant change in synaptic charge transfer was observed (Fig. 7B and C). Consistent with the notion that the rate of vesicle recycling is increased at physiological temperature (Pyott and Rosenmund, 2002), we found that depression of synchronous component of IPSCs triggered by trains of action potentials was slower at $35^{\circ} \mathrm{C}$ than at $25^{\circ} \mathrm{C}$ (Fig. $7 \mathrm{E}$ and F).
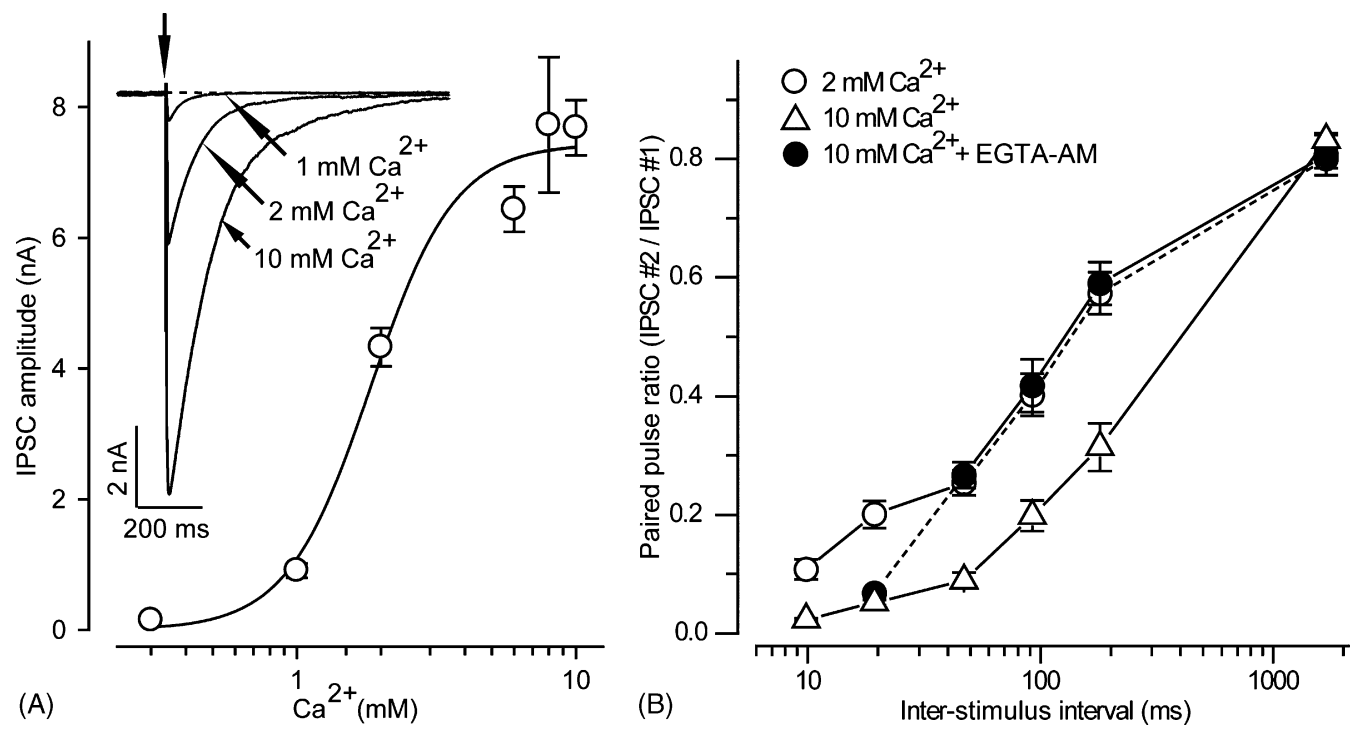

Fig. 5. Calcium-dependence and paired-pulse depression of IPSCs in cortical cultures. (A) Average amplitudes of IPSCs triggered by single action potentials and monitored in various concentration of free extracellular calcium. Data were fitted to a Hill function (see text; $\mathrm{EC}_{50}=1.8 \pm 0.2 \mathrm{mM}\left[\mathrm{Ca}^{2+}\right]_{\mathrm{o}} ; \mathrm{Hill}_{\mathrm{coefficient} 2.9}$ $n \geq 11$ ). Inserts illustrate representative traces of IPSCs recorded in 1,2 and $10 \mathrm{mM}$ of free extracellular $\mathrm{Ca}^{2+}$ as indicated by arrows. Scale bars apply to all traces. (B) Paired pulse depression of IPSCs monitored in $2 \mathrm{mM}(n=20)$ or $10 \mathrm{mM}(n=7)$ extracellular calcium $\mathrm{Ca}^{2+}$, or $10 \mathrm{mM}$ extracellular $\mathrm{Ca}^{2+}$ with addition of $0.1 \mathrm{mM}$ membrane-permeable calcium chelator EGTA-AM $(n=4)$. Average paired-pulse IPSC ratios were plotted a function of inter-stimulus interval between the first and second action potentials. 


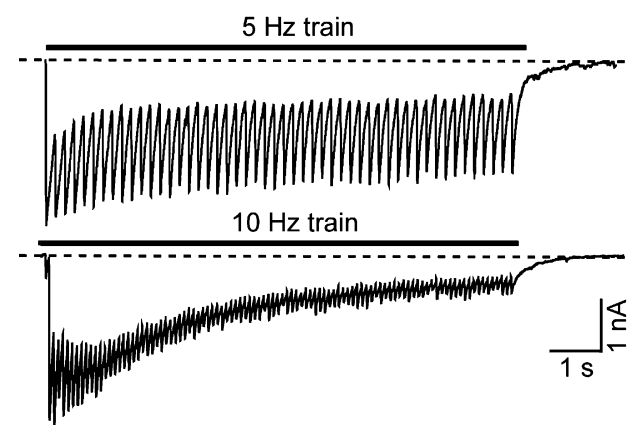

(A)

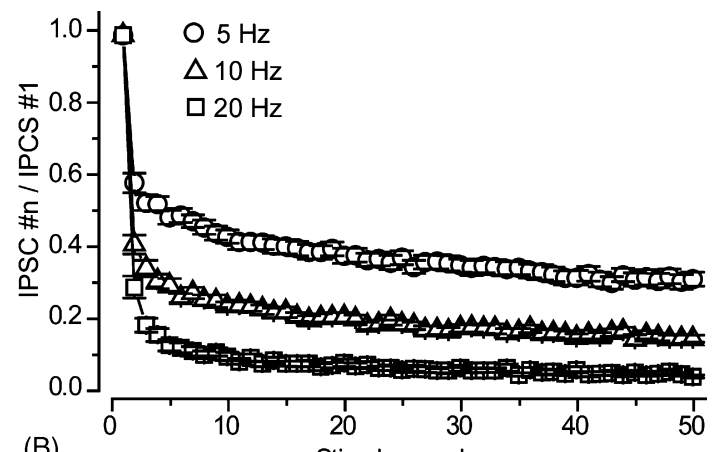

(B)

Stimulus number

Fig. 6. Depression of synchronous IPSCs triggered by high-frequency trains. (A) Typical examples of IPSCs triggered by high-frequency stimulus trains of action potentials applied for $10 \mathrm{~s}$ at $5 \mathrm{~Hz}$ (top trace) and $10 \mathrm{~Hz}$ (bottom trace). The stimulus artifacts were removed. Scale bars apply to both traces. (B) Depression of synchronous IPSCs during the trains of 50 action potentials applied at 5,10 and $20 \mathrm{~Hz}$. The amplitudes of individual synchronous IPSCs were normalized to the amplitude of first IPSC in the train, and plotted as a function of stimulus number. All recordings were performed in $2 \mathrm{mM}$ extracellular calcium. For each group $n \geq 10$.
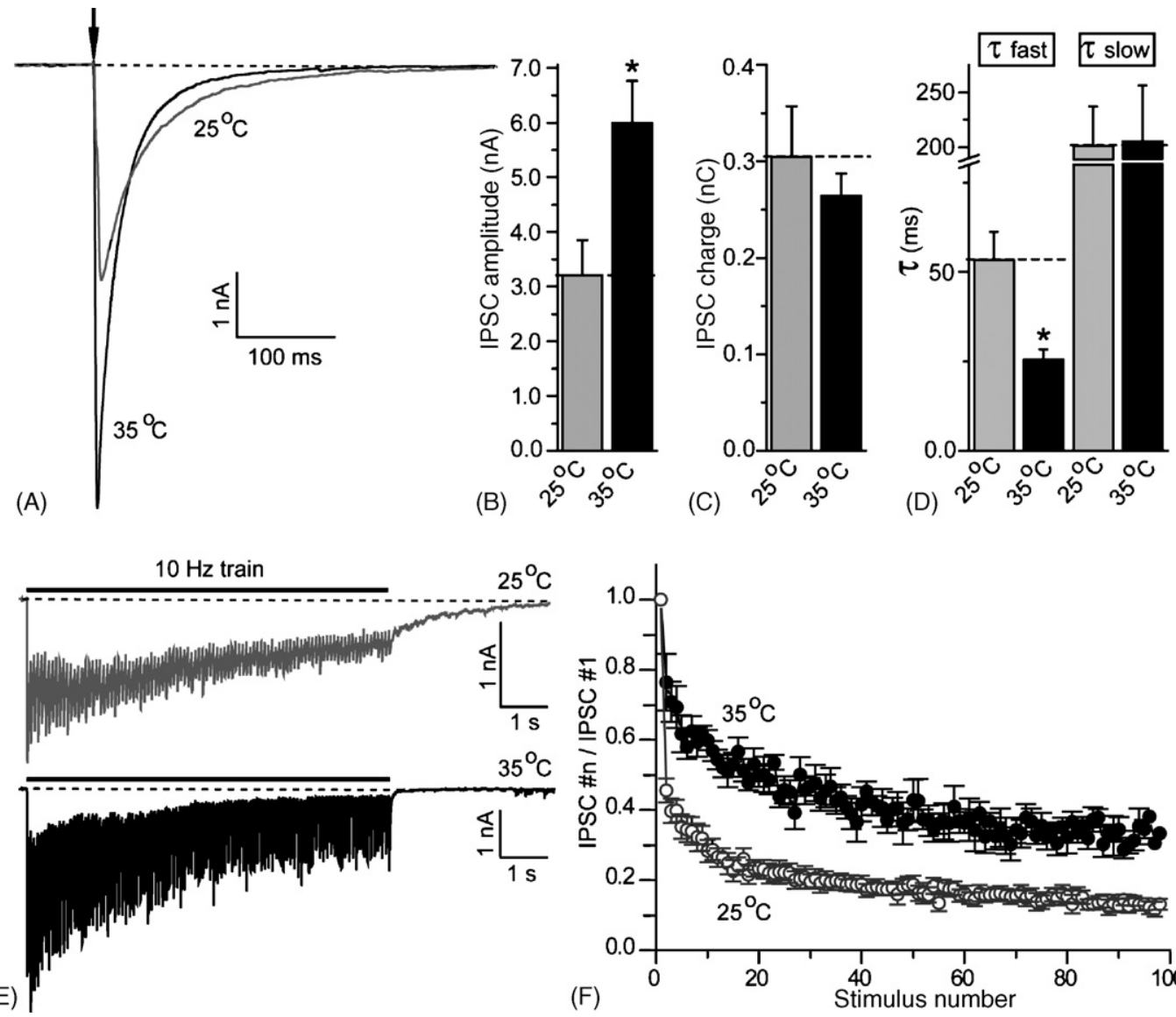

(E)

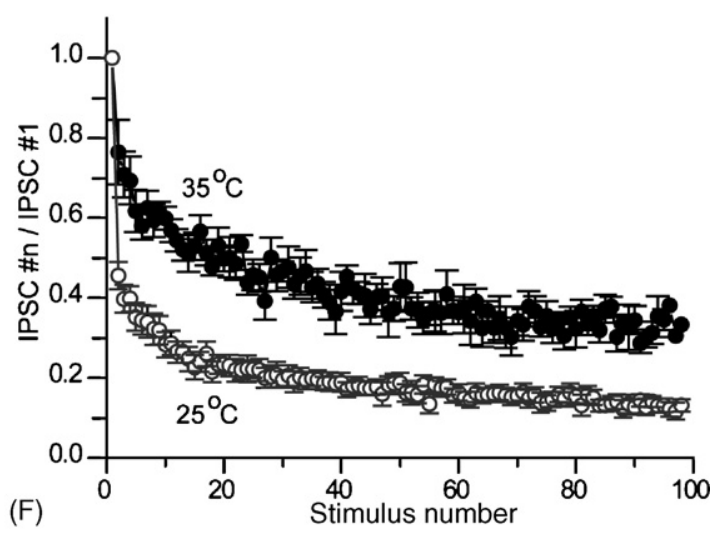

Fig. 7. Temperature dependence of transmitter release in inhibitory synapses. IPSCs were recorded in $2 \mathrm{mM}$ extracellular calcium with a 25 or $35^{\circ} \mathrm{C}$ bath temperature. (A) Typical examples of individual IPSCs recorded at $25^{\circ} \mathrm{C}$ (shown in grey) or $35^{\circ} \mathrm{C}$ (shown in black). Scale bars apply to both traces. (B and C) Average amplitudes (B) and charge transfers (C) of IPSCs monitored at $25^{\circ} \mathrm{C}(n=5)$ or $35^{\circ} \mathrm{C}(n=7)$. (D) Average time constants for fast ( $\tau$ fast) and slow ( $\tau$ slow) components of IPSCs monitored at 25 or $35^{\circ} \mathrm{C}$ were determined by fitting the plots of cumulative charge transferred during individual IPSCs to a double-exponential function (asterisk $=p<0.001$ ). (E) Typical examples of IPSCs triggered at $25^{\circ} \mathrm{C}$ (top trace, shown in grey) or $35^{\circ} \mathrm{C}$ (bottom trace, shown in black) by trains of action potentials applied at $10 \mathrm{~Hz}$. (F) Depression of synchronous IPSCs monitored at 25 or $35^{\circ} \mathrm{C}$ during the trains of 100 action potentials applied at $10 \mathrm{~Hz}, 25^{\circ} \mathrm{C}(n>20) ; 35^{\circ} \mathrm{C}$ $(n=7)$. 


\subsection{Excitatory synaptic transmission in cortical neurons}

Excitatory postsynaptic currents (EPSCs) can be pharmacologically isolated in cortical cultures by blocking $\mathrm{GABA}_{\mathrm{A}}$ receptors with bicuculline or picrotoxin (Fig. 1C). When postsynaptic neurons are maintained at a negative holding potential $(-70 \mathrm{mV})$, glutamatergic EPSCs have an inward direction and are primarily mediated by AMPA receptors because NMDA receptors are blocked by the extracellular $\mathrm{Mg}^{2+}$ under these conditions (Fig. 8A). Switching the holding potential of postsynaptic neurons to $+40 \mathrm{mV}$ relieves NMDA receptors from the $\mathrm{Mg}^{2+}$ block. Under these conditions, both AMPA and NMDA receptors contribute to the postsynaptic responses that have an outward direction. Because of significant difference in the kinetics of decay, the magnitudes of AMPA and NMDA currents can be estimated from the same outward EPSCs by measuring the maximum current amplitude immediately after stimulation (for fast AMPA responses) and $50 \mathrm{~ms}$ after stimulation (for slow NMDA reponses; Fig. 8B). Such measurements of AMPA/NMDA ratios in the same neuron have been extensively used for studying AMPA receptor trafficking (Myme et al., 2003; Takahashi et al., 2003; Rouach et al., 2005), and can be well applied to high-density cultures. Analysis of the AMPA- and NMDA-receptor dependent component of the evoked EPSC in three different cortical cultures showed that the average amplitudes of excitatory synaptic responses ranged between $1.5 \pm 0.2$ and $1.7 \pm 0.2 \mathrm{nA}$ for AMPA EPSCs and $0.8 \pm 0.1$ and $1.1 \pm 0.2 \mathrm{nA}$ for NMDA EPSCs (Fig. 8C). Thus similar to IPSCs, the magnitude of EPSCs is reproducible from culture to culture. In addition, we found that the rate of depression of AMPA EPSCs triggered by high frequency trains of action potentials (Fig. 8G and H) was similar to excitatory synapses of autaptic
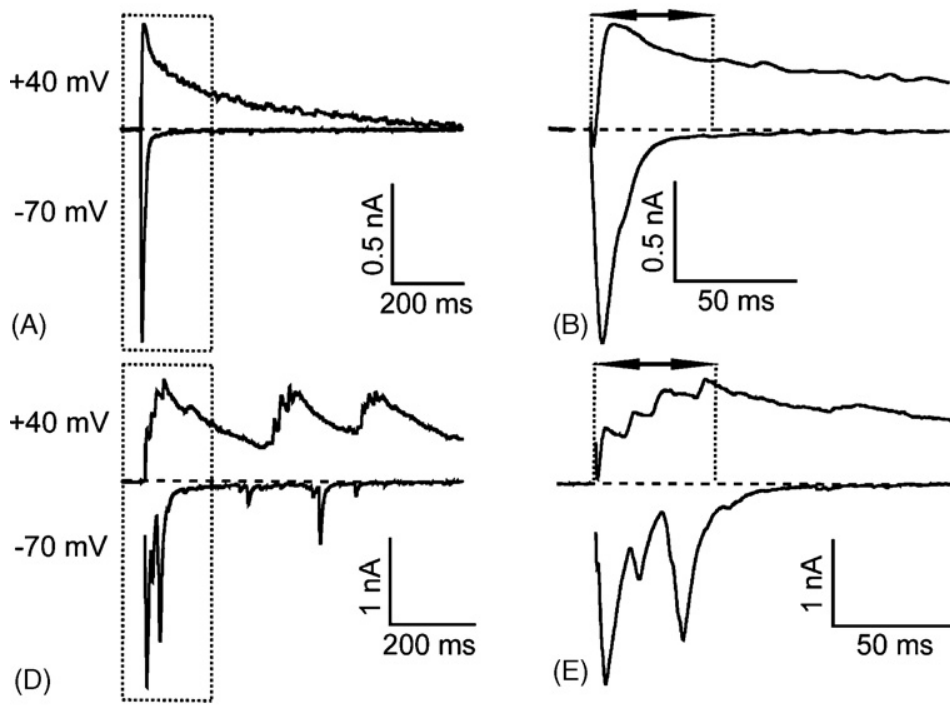

(B)

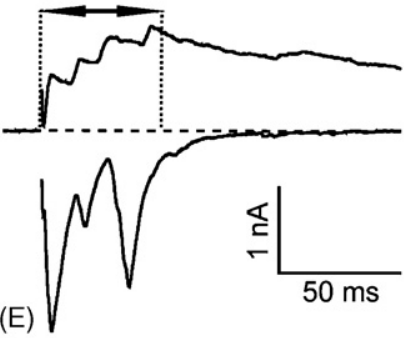

(C)

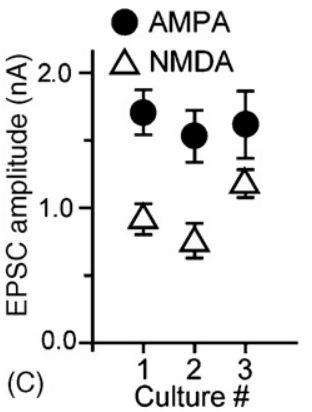

(F)

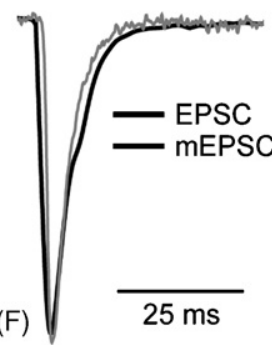

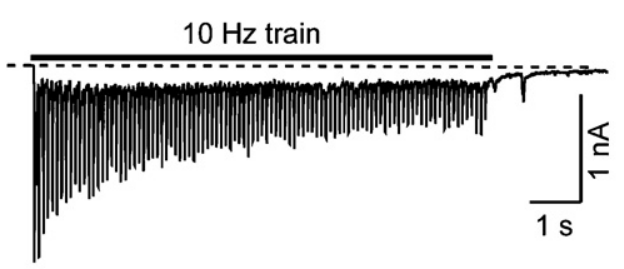

$(\mathrm{G})$

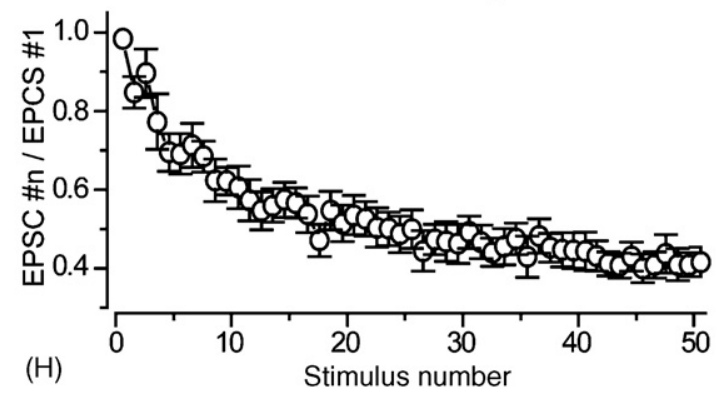

Fig. 8. Excitatory synaptic transmission in cortical neurons. Excitatory postsynaptic currents (EPSCs) were recorded in the presence of GABA receptor blocker picrotoxin (PTX, $50 \mu \mathrm{M}$ ) at holding potential of $-70 \mathrm{mV}$ to monitor AMPA EPSCs, or of $+40 \mathrm{mV}$ to monitor NMDA EPSCs. (A and B) Representative monosynaptic AMPA and NMDA EPSCs triggered by single action potentials applied at $0.1 \mathrm{~Hz}$. (B) Expansion of the area that is boxed in panel A to illustrate the analysis of NMDA EPSCs. Arrows indicate the $50 \mathrm{~ms}$ windows used for analysis of NMDA EPSC amplitudes. The stimulus artifacts were removed. (C) Average amplitudes of AMPA and NMDA EPSCs observed in three different cortical cultures. For each culture data were collected at 14 and 15 DIV ( $n=8-14)$. (D and E) Representative polysynaptic AMPA and NMDA EPSCs triggered by single action potentials applied at $0.1 \mathrm{~Hz}$. (E) Expansion of the area that is boxed in panel D to illustrate the ease with which AMPA-receptor dependent EPSCs can still be analyzed in the case of polysynaptic EPSCs, whereas NMDA-receptor dependent EPSCs cannot. The stimulus artifacts were removed. Note that only the initial AMPA and NMDA EPSCs but not the subsequent EPSCs observed in the same neuron are synchronized with each other and times of stimulus application. (F) A scaled monosynaptic AMPA EPSC (shown in black) was superimposed with the scaled trace of AMPA mEPSC averaged from 40 individual mEPSC events (shown in grey). ( $\mathrm{G}$ and $\mathrm{H}$ ) Depression in AMPA EPSCs during $10 \mathrm{~Hz}$ trains of action potentials. (G) Representative trace of excitatory responses triggered by 100 action potentials applied at $10 \mathrm{~Hz}$ and recorded at $-70 \mathrm{mV}$. The stimulus artifacts were removed. $(\mathrm{H})$ The amplitudes of individual EPSCs during the stimulus train, normalized to the amplitude of first response and plotted a function of stimulus number ( $n=15$ neurons from two independent cultures). 
hippocampal cultures (Pyott and Rosenmund, 2002; Otsu et al., 2004).

A possible concern about this measurement approach is that in the presence of GABA receptor blockers, cortical neurons frequently display network activity due to spontaneous firing of action potentials (data not shown). Activation of cascades of excitatory neurons may result in contamination of evoked EPSCs by 'multisynaptic' responses that exhibit a significant delay and are superimposed onto the EPSCs initiated immediately after stimulus application. Indeed, we observed two distinct types of EPSCs when synaptic transmission was triggered by single action potentials applied at $0.1 \mathrm{~Hz}$, and monitored in the presence of picrotoxin (PTX, $50 \mu \mathrm{M}$ ) in the bath solution. 'Monosynaptic' responses recorded from the same neuron maintained at $-70 \mathrm{mV}$ (to monitor AMPA EPSCs) or $+40 \mathrm{mV}$ (to monitor NMDA EPSCs) appeared as single peaks of current rapidly decaying to the baseline (Fig. 8A and B). The decay kinetics of the AMPA EPSCs observed in these neurons was similar to the kinetics of spontaneous miniature AMPA currents (mEPSCs), indicating that evoked responses were indeed 'monosynaptic' (Fig. 8F). In contrast, 'polysynaptic' responses were contaminated with secondary peaks of EPSCs appearing with a delay, most likely because local networks were activated (Fig. 8D and E). The presence of a contaminating network responses does not

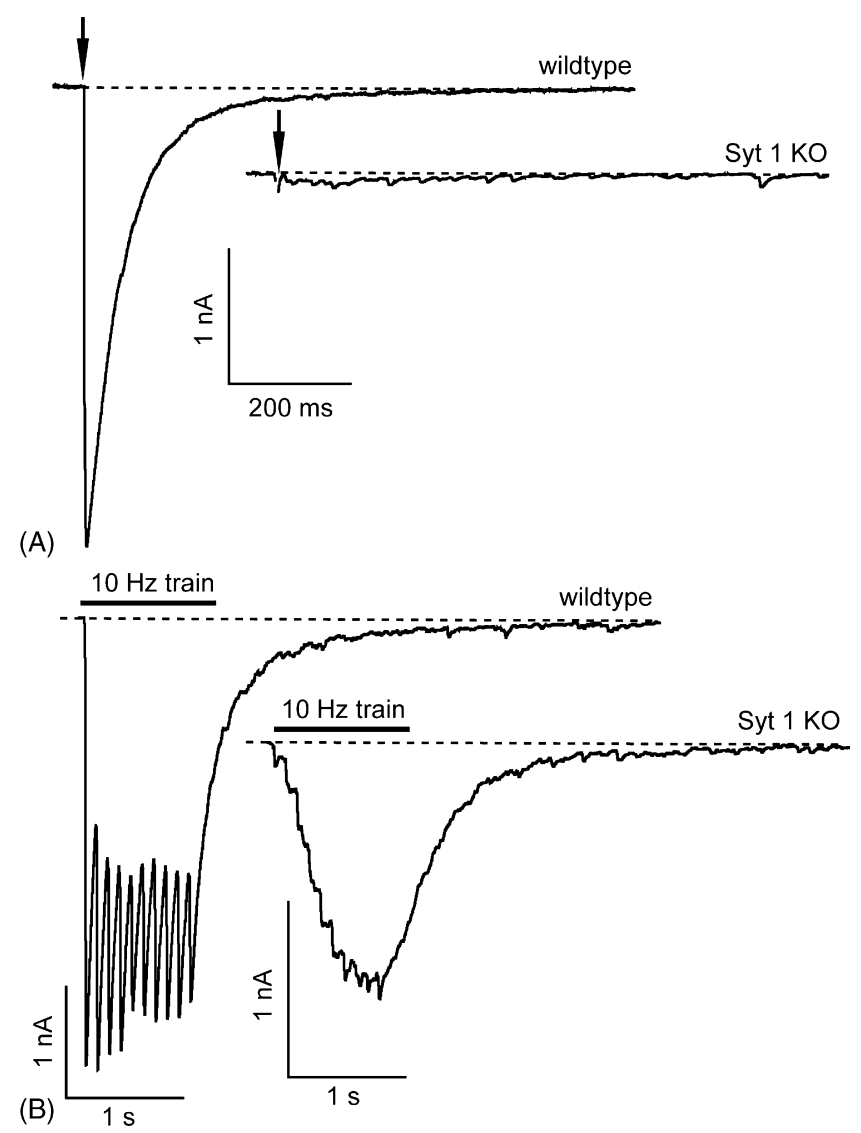

Fig. 9. Representative IPSCs monitored from wildtype of synaptotagmin-1 deficient (Syt $1 \mathrm{KO}$ ) neurons in $2 \mathrm{mM}$ extracellular calcium. IPSCs were triggered by single action potentials (A) or trains of 10 action potentials applied at $10 \mathrm{~Hz}$ (B). The stimulus artifacts were removed. See Maximov and Südhof (2005) for a more extensive description of the phenotype. affect the measurements of AMPA EPSC amplitudes (because of the fast kinetics of AMPA currents), but makes it difficult to analyze the amplitudes of NMDA EPSCs. Therefore, the neurons that displayed contaminating network responses during the first $50 \mathrm{~ms}$ after stimulation could not be used for analysis of NMDA EPSC amplitudes and AMPA/NMDA ratios.

\subsection{Inhibitory synaptic transmission in neurons lacking synaptotagmin-1}

Fast synaptic vesicle exocytosis is triggered by $\mathrm{Ca}^{2+}$-binding to synaptotagmin-1 (Fernandez-Chacon et al., 2001). Deletion of synaptotagmin-1 results in a loss of synchronous component of neurotransmitter release in excitatory (Geppert et al., 1994; Nishiki and Augustine, 2004) and inhibitory (Maximov and Südhof, 2005) synapses. Consistent with our previous observations, we found that synchronous IPSCs triggered by single action potentials were suppressed in neurons from synaptotagmin-1 knock-out mice (Fig. 9A). The remaining asynchronous release was small when synaptotagmin-1 deficient neurons were stimulated at low frequency but become predominant during the trains of action potentials (Fig. 9B).

\section{Discussion}

In this study, we describe a method to analyze inhibitory and excitatory synaptic transmission in mixed high-density cultures of neurons. A major problem in analyzing synaptic transmission in high-density cultures of neurons - as opposed to isolated neurons that are cultured on glial microislands and that form autapses - has been the difficulty of reliably stimulating presynaptic inputs in such a manner that postsynaptic responses can be recorded with reproducible properties and amplitudes. We demonstrate that a combination of extracellular stimulation of presynaptic inputs via a local bipolar electrode and postsynaptic whole-cell voltage-clamp recording allows monitoring both excitatory and inhibitory synaptic transmission. In principle, the approach is similar to those used by others previously, and fundamentally represents a method that lies in between paired recordings as the most accurate but also labor-intensive technique, and field stimulation as the simplest but most artifact prone technique. The usefulness of the approach we describe rests on its ability to achieve reproducible measurements of amplitudes that are similar from culture to culture.

Using IPSCs as an example, we show that in the recording configuration we use, stimulation of presynaptic inputs with an extracellular bipolar electrode is dependent on the strength and duration of the stimulus. At low stimulus strengths or short stimulus durations, postsynaptic responses are small and variable. Increasing stimulus strengths and durations leads to a saturation of the response at a plateau, and a decrease in response variability (Fig. 3). These results indicate that postsynaptic responses induced by the electrode stimuli are mediated by release of neurotransmitter in a population of synapses formed by several axons of surrounding neurons. The number of synapses that are stimulated with a saturating pulse seem to be fairly constant, independent of the culture because under our conditions, 
the amplitudes obtained were relatively reproducible between cultures obtained even from different investigators (Fig. 4). We also demonstrate that the magnitude and kinetics of IPSCs triggered by single action potentials or action potential trains depend on the extracellular calcium concentration, intracellular calcium buffering, temperature, and stimulation frequency, suggesting that inhibitory synapses triggered by local bipolar electrodes display a normal calcium sensitivity and short-term plasticity (Figs. 5 and 6). It is important to ensure in all of these experiments that the presynaptic stimulus that is applied close to the postsynaptic neuron does not cause artifacts in the postsynaptic recordings; however, an analysis of the stimulus artifact (Fig. 2) clearly ruled out this possibility.

When compared to other stimulation methods, triggering synaptic transmission by local electrodes has several advantages. Stimulation with local electrodes does not require labor-intensive screening for synaptically connected neuronal pairs, and therefore enables rapid and efficient analysis of the effects of pharmacological agents, protein expression and genetic manipulations on neurotransmitter release. Unlike double patch techniques (Bi and Poo, 1998) or whole-cell recordings from autaptic microisland cultures (Bekkers and Stevens, 1991), triggering action potentials with extracellular electrodes enables monitoring synaptic transmission in near-physiological conditions when presynaptic neurons are not affected by mechanical penetration and/or by wash-out of intracellular components due to dialysis of the whole-cell pipette solution. Moreover, the stimulation of multiple inputs monitors a larger population of synaptic connections simultaneously. In contrast to massive field stimulations, stimulation with local electrodes does not produce large stimulus artifacts that may contaminate postsynaptic signals (Fig. 3C and D), because local action potential triggering can be achieved by injection of a relatively small current. Synaptic responses triggered by local electrodes saturate at stimulus intensity of $<0.9 \mathrm{nA}$ for a $1 \mathrm{~ms}$ pulse, whereas up to $30 \mathrm{~mA}$ stimulations have been used with some field electrodes (Schoch et al., 2001; Deak et al., 2004). Finally, local stimulations only affect a small area of a neuronal culture dish, making it possible to record from multiple neurons in the same preparation without producing the use-dependent changes in all neurons.

The basic properties of synaptic transmission we describe here are similar to those characterized in other culture and slice preparations. First, we show that increasing the concentration of free extracelluar calcium results in augmentation of inhibitory synaptic responses (Fig. 5), which is a well-established aspect of synaptic release (Katz and Miledi, 1968; Hille, 2001). Second, the short-term plasticity of inhibitory synapses monitored in cortical neurons stimulated by high frequency trains of action potentials (Fig. 6) is similar to the properties of inhibitory synapses observed in acute slices (Varela et al., 1999). Finally, synaptic responses recorded from cortical neurons lacking calcium sensor for synaptic vesicle exocytosis, synaptotagmin-1, are similar to those obtained from autaptic cultures (Fig. 9; Geppert et al., 1994; Maximov and Südhof, 2005). Thus, monitoring synaptic transmission in high-density cultures of neurons triggered by local stimulation is a valuable tool for studies of various mechanisms of synaptic transmission.

\section{Conclusion}

Here, we describe a method to monitor inhibitory and excitatory synaptic transmission in mixed high-density cultures of neurons from different brain areas that allows reproducible and facile analysis of synaptic responses. Our approach combines local stimulation of presynaptic axons with a bipolar electrode with conventional whole-cell recordings of postsynaptic neurons. Our data indicate that stimulation via local bipolar electrodes enables rapid and efficient analysis of synaptic phenotypes in cultured neurons in near-physiological conditions. This stimulation method can be used for drug screening and studies of regulation of neurotransmitter release in wildtype neurons infected with lentiviruses that express various components effecting synaptic vesicle release machinery and neurons from genetically modified mice.

\section{Acknowledgements}

We wish to thank I. Kornblum, N. Hamlin, J. Mitchell, and L. Fan for excellent technical assistance. This study was supported in part by NIH grants R37-MH52804-06 and R37-MH5280408, HHMI (D.G.R.T. and T.C.S.), George A. and Marjorie H. Anderson Fellowship (D.G.R.T.).

\section{References}

Bekkers JM, Stevens CF. Excitatory and inhibitory autaptic currents in isolated hippocampal neurons maintained in cell culture. Proc Natl Acad Sci USA 1991;88(17):7834-8.

Bi GQ, Poo MM. Synaptic modifications in cultured hippocampal neurons: dependence on spike timing, synaptic strength, and postsynaptic cell type. J Neurosci 1998;18(24):10464-72.

Chen G, Harata NC, Tsien RW. Paired-pulse depression of unitary quantal amplitude at single hippocampal synapses. Proc Natl Acad Sci USA 2004;101(4):1063-8.

Deak F, Schoch S, Liu X, Südhof TC, Kavalali ET. Synaptobrevin is essential for fast synaptic-vesicle endocytosis. Nat Cell Biol 2004;6: 1102-8.

Fernandez-Chacon R, Konigstorfer A, Gerber SH, Garcia J, Matos MF, Stevens CF, Brose N, Rizo J, Rosenmund C, Südhof TC. Synaptotagmin I functions as a calcium regulator of release probability. Nature 2001;410: 41-9.

Geppert M, Goda Y, Hammer RE, Li C, Rosahl TW, Stevens CF, Südhof TC. Synaptotagmin I: a major $\mathrm{Ca}^{2+}$ sensor for transmitter release at a central synapse. Cell 1994;79:717-27.

Hamill OP, Marty A, Neher E, Sakmann B, Sigworth FJ. Improved patch-clamp techniques for high-resolution current recording from cells and cell-free membrane patches. Pflugers Arch 1981;391:85-100.

Hille B. Voltage-gated calcium channels. In: Ion channels of excitable membranes. Sunderland, Massachusetts, USA: Sinauer Associates Inc; 2001. p. 95-129.

Ho A, Morishita W, Atasoy D, Liu X, Tabuchi K, Hammer RE, Malenka RC and Südhof TC. Genetic analysis of Mint/X11 proteins: essential presynaptic functions of a neuronal adaptor protein family, submitted for publication.

Katz B, Miledi R. The role of calcium in neuromuscular facilitation. J Physiol 1968;195:481-92.

Kirischuk S, Grantyn R. Intraterminal $\mathrm{Ca} 2+$ concentration and asynchronous transmitter release at single GABAergic boutons in rat collicular cultures. J Physiol 2003;548(Pt. 3):753-64.

Kirischuk S, Veselovsky N, Grantyn R. Single-bouton-mediated synaptic transmission: postsynaptic conductance changes in their relationship with presynaptic calcium signals. Pflügers Arch 1999;438:716-24. 
Liu G, Tsien RW. Synaptic transmission at single visualized hippocampal boutons. Neuropharmacology 1995a;1995(11):1407-21.

Liu G, Tsien RW. Properties of synaptic transmission at single hippocampal synaptic boutons. Nature 1995b;375(6530):404-8.

Maximov A, Südhof TC. Autonomous function of synaptotagmin-1 1 in triggering asynchronous release independent of asynchronous release. Neuron 2005;48:547-54.

Myme CI, Sugino K, Turrigiano GG, Nelson SB. The NMDA-to-AMPA ratio at synapses onto layer $2 / 3$ pyramidal neurons is conserved across prefrontal and visual cortices. J Neurophysiol 2003;90(2):771-9.

Nishiki T, Augustine GJ. Synaptotagmin I synchronizes transmitter release in mouse hippocampal neurons. J Neurosci 2004;24(27):6127-32.

Otsu Y, Shahrezaei V, Li B, Raymond LA, Delaney KR, Murphy TH. Competition between phasic and asynchronous release for recovered synaptic vesicles at developing hippocampal autaptic synapses. J Neurosci 2004;24(2):420-33.

Pang ZP, Sun J, Rizo J, Maximov A, Südhof TC. Genetic analysis of synaptotagmin-2 in spontaneous and $\mathrm{Ca}(2+)$-triggered neurotransmitter release. EMBO J 2006a;25:2039-50.

Pang ZP, Shin OH, Meyers A, Rosenmund C, Südhof TC., 2006b. A gainof-function mutation in synaptotagmin-1 reveals a critical role of $\mathrm{Ca} 2+-$ dependent SNARE-complex binding in synaptic exocytosis, J Neurosci, in press.
Pyott SJ, Rosenmund C. The effects of temperature on vesicular supply and release in autaptic cultures of rat and mouse hippocampal neurons. J Physiol 2002;539(Pt. 2):523-35.

Rouach N, Byrd K, Petralia RS, Elias GM, Adesnik H, Tomita S, Karimzadegan S, Kealey C, Bredt DS, Nicoll RA. TARP gamma-8 controls hippocampal AMPA receptor number, distribution and synaptic plasticity. Nat Neurosci 2005;8(11):1525-33.

Schoch S, Deak F, Konigstorfer A, Mozhayeva M, Sara Y, Südhof TC, Kavalali ET. SNARE function analyzed in synaptobrevin/VAMP knockout mice. Science 2001;2001(294):1117-22.

Swanwick CC, Murthy NR, Mtchedlishvili Z, Sieghart W, Kapur J. Development of gamma-aminobutyric acidergic synapses in cultured hippocampal neurons. J Comp Neurol 2006;495(5):497-510.

Takahashi T, Svoboda K, Malinow R. Experience strengthening transmission by driving AMPA receptors into synapses. Science 2003;299(5612):1585-8.

Tang J, Maximov A, Shin OH, Dai H, Rizo J, Südhof TC. A complexin/ synaptotagmin-1 switch controls fast synaptic vesicle exocytosis. Cell 2006;126(6):1175-87.

Varela JA, Song S, Turrigiano GG, Nelson SB. Differential depression at excitatory and inhibitory synapses in visual cortex. J Neurosci 1999;19(11): 4293-304.

Zucker RS, Regehr WG. Short-term synaptic plasticity. Annu Rev Physiol 2002;64:355-405. 